\title{
Atypical Residual Bodies
}

National Cancer Institute

\section{Source}

National Cancer Institute. Atypical Residual Bodies. NCI Thesaurus. Code C120866.

Abnormally large, misshapen and/or clumped vacuoles containing cell debris in the testis,

or present in stages of spermatogenesis when not normally seen. 